$\mathrm{J}$ o u r n a l of

Mathematics

and Applications

JMA No 43, pp 113-121 (2020)

\title{
Boolean Algebra of One-Point Local Compactifications
}

\author{
Artur Polański
}

ABstract: For a given locally compact Hausdorff space we introduce a Boolean algebra structure on the family of all its one-point local compactifications.

AMS Subject Classification: 54D45, 03G05.

Keywords and Phrases: Local; Compactification; Boolean; Algebra; Ends.

\section{Introduction}

Every locally compact, noncompact Hausdorff space $X$ has a well known one-point compactification (Alexandroff compactification, [1]). In this paper we consider the set $\mathcal{B}(X)$ of all one-point local compactifications of $X$ up to an equivalence. We prove that $\mathcal{B}(X)$ is a partially ordered set such that the order $\leqslant$ induces a Boolean algebra. Moreover, the elements 0 and 1 of $\mathcal{B}(X)$ are respectively $X$ and $\omega X$. Then we focus on describing the algebra we get using topological notions and convergence and we provide examples by computing the algebra in some special cases. We also note the connection with the topic of ends of manifolds (see [2, pages 110-118]), as for a noncompact, connected, second countable manifold $L$ with $n$ ends, $n<\infty$, we have $|\mathcal{B}(L)|=2^{n}$.

\section{Notation and terminology}

- Throughout the paper, ZFC is assumed.

- Given a locally compact Hausdorff space $X$ we denote by $\omega X$ a one-point compactification of $X$ if $X$ is not compact and $X$ otherwise,

COPYRIGHT @ by Publishing House of Rzeszów University of Technology P.O. Box 85, 35-959 Rzeszów, Poland 
- a clopen set is a set that is both closed and open,

- if $Y$ is a one-point local compactification different from $X$, the unique point of $Y \backslash X$ will be denoted by $\infty_{Y}$,

- a filter $\mathcal{F}$ of open sets in a topological space $X$ is a non-empty family of sets open in $X$ such that $\emptyset \notin \mathcal{F}$ and, for all $V_{1}, V_{2} \in \mathcal{F}$ and an open $V \subset X$ we have $V_{1} \cap V_{2} \in \mathcal{F} \Rightarrow V \in \mathcal{F}$.

\section{Main results}

Definition 1. If $X$ is a locally compact Hausdorff space, we call $(Y, f)$ an at most onepoint local compactification of $X$ iff $Y$ is a locally compact Hausdorff and $f: X \rightarrow Y$ is a homeomorphic embedding such that $f(X)$ is dense in $Y$ and $|Y \backslash f(X)| \leqslant 1$. If $(Y, f)$ is an at most one point local compactification of $X$ and $|Y \backslash f(X)|=1$, we call $(Y, f)$ a one-point local compactiication of $X$.

For simplicity, we say that $Y$ is a/an (at most) one-point local compactification of $X$ iff $\left(Y, \mathrm{id}_{X}\right)$ is a/an (at most) one-point local compactification of $X$.

Definition 2. Let $X$ be a locally compact Hausdorff space, $\left(Y_{1}, f_{1}\right)$ and $\left(Y_{2}, f_{2}\right)$ its at most one-point local compactifications. We will write $\left(Y_{1}, f_{1}\right) \leqslant\left(Y_{2}, f_{2}\right)$ (or, for simplicity, $\left.Y_{1} \leqslant Y_{2}\right)$ iff one of the following conditions apply:

- $f_{1}(X)=Y_{1}$

- $Y_{1}=f_{1}(X) \cup\left\{\infty_{Y_{1}}\right\}, Y_{2}=f_{2}(X) \cup\left\{\infty_{Y_{2}}\right\}$ and the function

$$
Y_{1} \ni x \mapsto\left\{\begin{array}{ll}
f_{2}\left(f_{1}^{-1}(x)\right), & x \in f_{1}(X) \\
\infty_{Y_{2}}, & x=\infty_{Y_{1}}
\end{array} \in Y_{2}\right.
$$

is continuous.

Note that $\leqslant$ is reflexive and transitive, with $0=X$ and $1=\omega X$. We can define an equivalence relation $\equiv$ by

$$
\left(Y_{1}, f_{1}\right) \equiv\left(Y_{2}, f_{2}\right) \text { iff }\left(Y_{1}, f_{1}\right) \leqslant\left(Y_{2}, f_{2}\right) \text { and }\left(Y_{2}, f_{2}\right) \leqslant\left(Y_{1}, f_{1}\right),
$$

or, for simplicity,

$$
Y_{1} \equiv Y_{2} \text { iff } Y_{1} \leqslant Y_{2} \text { and } Y_{2} \leqslant Y_{1}
$$

We also define

$$
\mathcal{B}(X):=\{Y \text {-one-point local compactification of } X\} / \equiv .
$$

From now on instead of an equivalence class of $Y$ in $\mathcal{B}(X)$ we will just write $Y$.

We are now ready to state the first result where we will prove that $\mathcal{B}(X)$ ordered by $\leqslant$ is a Boolean algebra, by showing that it is in fact order isomorphic to a much simpler one. 
Theorem 1. Given a locally compact Hausdorff space $X, \mathcal{B}(X)$ is a partially ordered space with a lattice such that the order $\leqslant$ induces a Boolean algebra, i.e., for $Y_{1}, Y_{2}$ one-point local compactifications of $X$ :

- $Y_{1} \vee Y_{2}=\sup _{\leqslant}\left\{Y_{1}, Y_{2}\right\}$,

- $Y_{1} \wedge Y_{2}=\inf _{\leqslant}\left\{Y_{1}, Y_{2}\right\}$,

- $0=X$

- $1=\omega X$

- for any space $Y \in \mathcal{B}(X)$ there exists a unique space $\backslash Y \in \mathcal{B}(X): Y \wedge \backslash Y=0$, $Y \vee \backslash Y=1$.

In particular, $0=1$ iff $X$ is compact.

Proof. First consider $\beta X$, a Čech-Stone compactification of $X$. We define $\mathcal{A}(X):=$ $\{F \subset \beta X \backslash X: F$ clopen in $\beta X \backslash X\}$ (note that $\beta X \backslash X$ is compact). $\mathcal{A}(X)$ with standard set operations is a Boolean algebra. We will show an isomorphism between $\mathcal{B}(X)$ and $\mathcal{A}(X)$, proving that $\mathcal{B}(X)$ is also a Boolean algebra.

To this end, we will define $f: \mathcal{B}(X) \rightarrow \mathcal{A}(X)$. If $X$ is compact, both $\mathcal{B}(X)$ and $\mathcal{A}(X)$ are trivial, therefore assume that $X$ is not compact. Consider a clopen in $\beta X \backslash X$ set $F$ such that $\emptyset \neq F \neq \beta X \backslash X$. We can now identify $F$ and $(\beta X \backslash X) \backslash F$ with points, getting a compact space $X \cup\{\{F\}\} \cup\{\{(\beta X \backslash X) \backslash F\}\}$. Its subspace $X \cup\{\{F\}\}$ is then a one-point local compactification of $X$. Conversely, for any onepoint local compactification $Y$ of $X$ there exists a unique clopen in $\beta X \backslash X$ set $F_{Y}$ such that $Y$ is equivalent with $X \cup\left\{\left\{F_{Y}\right\}\right\}$ (from the universal property of $\beta X$ ). We define $f(X)=\emptyset$ and for every one-point local compactification $Y$ of $X$ we put $f(Y)=F_{Y}$, where $Y$ is the unique clopen in $\beta X \backslash X$ set such that $Y$ is equivalent to $X \cup\left\{\left\{F_{Y}\right\}\right\}$. It can be easily seen that for one-point local compactifications $Y_{1}, Y_{2}$ of $X$ we have $Y_{1} \leqslant Y_{2}$ iff $F_{Y_{1}} \subset F_{Y_{2}}$, so $f$ preserves the partial order and is indeed an isomorphism. Furthermore, for one-point local compactifications $Y_{1}, Y_{2}$ of $X$ we have:

1. $Y_{1} \vee Y_{2}=X \cup\left\{\left\{F_{Y_{1}} \cup F_{Y_{2}}\right\}\right\}$.

2. $Y_{1} \wedge Y_{2}=X \cup\left\{\left\{F_{Y_{1}} \cap F_{Y_{2}}\right\}\right\}$ if $F_{Y_{1}} \cap F_{Y_{2}} \neq \emptyset$ and $Y_{1} \wedge Y_{2}=X$ otherwise.

3. $\backslash Y=X \cup\left\{\left\{(\beta X \backslash X) \backslash F_{Y}\right\}\right\}$ for $\emptyset \neq F_{Y} \neq \beta X \backslash X$.

Remark 1. The proof of Theorem 1 shows that $\mathcal{B}(X)$ is isomorphic (as a Boolean algebra) to the algebra of all clopen subsets of the remainder $\beta X \backslash X$ of $X$. One easily concludes that the Stone space of $\mathcal{B}(X)$ is homeomorphic to the space of all connected components of $\beta X \backslash X$ (that is, the space obtained from $\beta X \backslash X$ by identifying points that lie in a common connected component). 
Now that we know that $\mathcal{B}(X)$ is a Boolean algebra, we will focus on describing it without using $\mathcal{A}(X)$. If we add a point $\left\{\infty_{Y}\right\}$ to a locally compact Hausdorff space $X$ to get its one-point local compactification $Y$, we only need to know the neighborhood basis at $\left\{\infty_{Y}\right\}$ to know its topology. To this end, let us introduce the following characterization. For simplicity, we will also use one more definition.

Definition 3. Let $X$ be a locally compact Hausdorff space, $Y$ its one-point local compactification. Then

$$
\tau(Y):=\left\{U \backslash\left\{\infty_{Y}\right\}: U \text { open neighborhood of } \infty_{Y} \text { in } Y\right\} .
$$

$\tau(Y)$ uniquely determines $Y \neq X, Y \in \mathcal{B}(X)$.

Proposition 1. Let $X$ be a locally compact Hausdorff space, $Y_{1}, Y_{2} \in \mathcal{B}(X), Y_{1}, Y_{2} \neq$ $0, Y_{1}, Y_{2} \neq 1$.

1. $\tau\left(Y_{1} \wedge Y_{2}\right)=\left\{U_{1} \cap U_{2}: U_{1} \in \tau\left(Y_{1}\right), U_{2} \in \tau\left(Y_{2}\right)\right\}$, provided that the sets $U_{1} \cap U_{2}$ are nonempty for all $U_{1} \in \tau\left(Y_{1}\right), U_{2} \in \tau\left(Y_{2}\right)$ and $Y_{1} \wedge Y_{2}=0$ otherwise.

2. $\tau\left(Y_{1} \vee Y_{2}\right)=\left\{U_{1} \cup U_{2}: U_{1} \in \tau\left(Y_{1}\right), U_{2} \in \tau\left(Y_{2}\right)\right\}=\tau\left(Y_{1}\right) \cap \tau\left(Y_{2}\right)$.

3. $\tau\left(\backslash Y_{1}\right)=\left\{X \backslash F: F \subset X\right.$, for any $U \in \tau\left(Y_{1}\right) F \backslash U$ compact $\}$.

Or, in terms of convergence:

(a) A net $\left(x_{\gamma}\right) \subset X$ in $Y_{1} \wedge Y_{2}$ is convergent to $\infty_{Y_{1} \wedge Y_{2}}$ iff $\left(x_{\gamma}\right)$ is convergent to $\infty_{Y_{1}}$ in $Y_{1}$ and to $\infty_{Y_{2}}$ in $Y_{2}$, and $Y_{1} \wedge Y_{2}=0$ if there is no such net.

(b) A net $\left(x_{\gamma}\right) \subset X$ in $Y_{1} \vee Y_{2}$ is convergent to $\infty_{Y_{1} \vee Y_{2}}$ iff every subnet of $\left(x_{\gamma}\right)$ has a subnet convergent to $\infty_{Y_{1}}$ in $Y_{1}$ or to $\infty_{Y_{2}}$ in $Y_{2}$.

(c) A net $\left(x_{\gamma}\right) \subset X$ in $\backslash Y_{1}$ is convergent to $\infty_{\backslash Y_{1}}$ iff $\left(x_{\gamma}\right)$ has no convergent subnets in $Y_{1}$.

Proof. Again, let $\beta X$ be a Čech-Stone compactification of $X$.

Note that if $Y$ is a one-point local compactification of $X$ and $F_{Y}$ is a clopen set in $\beta X \backslash X$ such that $Y$ is equivalent with $X \cup\left\{\left\{F_{Y}\right\}\right\}$, then

$$
\tau(Y)=\left\{X \cap U: U \supset F_{Y} \text { and } U \text { open in } \beta X\right\} .
$$

Following this notation consider $F_{Y_{1}}$ and $F_{Y_{2}}$ such that $Y_{1}$ and $Y_{2}$ are equivalent to $X \cup\left\{\left\{F_{Y_{1}}\right\}\right\}$ and $X \cup\left\{\left\{F_{Y_{2}}\right\}\right\}$ respectively.

Property (2) follows easily from $(*)$.

To see that $\left\{U_{1} \cup U_{2}: U_{1} \in \tau\left(Y_{1}\right), U_{2} \in \tau\left(Y_{2}\right)\right\}=\tau\left(Y_{1}\right) \cap \tau\left(Y_{2}\right)$, take any $U_{1} \in$ $\tau\left(Y_{1}\right), U_{2} \in \tau\left(Y_{2}\right)$. $U_{2}=\left(U_{2} \cup\left\{\infty_{Y_{2}}\right\}\right) \cap X$ is open in $X$, and thus open in $Y_{1}$. $U_{1} \cup\left\{\infty_{Y_{1}}\right\}$ is also open in $Y_{1}$ and thus so is $U_{1} \cup\left\{\infty_{Y_{1}}\right\} \cup U_{2}$. Similarly, $U_{1} \cup\left\{\infty_{Y_{2}}\right\} \cup U_{2}$ is open in $Y_{2}$. The reverse inclusion is trivial.

We turn to (1). If $F_{Y_{1}} \cap F_{Y_{1}}=\emptyset$ we have $Y_{1} \wedge Y_{2}=0$, assume the contrary. Consider $U$ open in $\beta X$ such that $F_{Y_{1}} \cap F_{Y_{1}} \subset U$ and take $V_{1}, V_{2}$ open in $\beta X$ such 
that $V_{1} \cap V_{2}=\emptyset$, and we have $F_{Y_{1}} \backslash U \subset V_{1}$ and $F_{Y_{2}} \backslash U \subset V_{2}$. Then $U_{1}:=V_{1} \cup U$ and $U_{2}:=V_{2} \cup U$ are open (in $\beta X$ ) supersets of respectively $F_{Y_{1}}$ and $F_{Y_{2}}$ such that $U_{1} \cap U_{2}=U$, which gives us (1).

We are left with (3). To see that

$$
\tau\left(\backslash Y_{1}\right) \subset\left\{X \backslash F: F \subset X \text {, for any } U \in \tau\left(Y_{1}\right) F \backslash U \text { compact }\right\},
$$

consider $V$ open in $\beta X$ such that $(\beta X \backslash X) \backslash F_{Y_{1}} \subset V$ and take any $U$ open in $\beta X$ such that $F_{Y_{1}} \subset U$. Then $(X \backslash V) \backslash U=X \backslash(U \cup V)=\beta X \backslash(U \cup V)$ is a closed subset of $\beta X$ contained in $X$ and therefore compact.

For the reverse inclusion, let $V_{0}$ and $W_{0}$ be open sets with disjoint closures in $\beta X$ such that $(\beta X \backslash X) \backslash F_{Y_{1}} \subset V_{0}$ and $F_{Y_{1}} \subset W_{0}$. Consider $F \subset X$ such that for any $U \in \tau\left(Y_{1}\right)$ the set $F \backslash U$ is compact. Take any $x \in X$ and its closed (in $X$ ) neighborhood $G$ such that $G$ is compact. Then $X \backslash G \in \tau\left(Y_{1}\right)$, so $F \cap G$ is compact. Since $x$ and its neighborhood $G$ were arbitrary, this implies that $F$ is closed in $X$ (since if we take $x$ from the boundary of $F$, we get that it must be in $F$ ). Similarly, since $F \cap \overline{V_{0}} \subset F \backslash W_{0}$ and $W_{0} \cap X \in \tau\left(Y_{1}\right)$, we get that $F \cap \overline{V_{0}}$ is compact which implies that $F \cup F_{Y_{1}}$ is closed in $\beta X$. Therefore we have $X \backslash F=X \cap\left(\beta X \backslash\left(F \cup F_{0}\right)\right) \in \tau\left(\backslash Y_{1}\right)$ which ends the proof of (3).

Properties (a) - (c) follow easily from (1) - (3).

On the other hand, one can wonder when a family $\mathcal{F}$ of sets open in a locally compact Hausdorff space $X$ induces its one-point local compactifiaction. The following proposition answers that question.

Proposition 2. Let $\mathcal{F}$ be a filter of open sets in a locally compact Hausdorff space $X$. Then $\mathcal{F}$ induces a one-point local compactification $Y$ of $X$ such that $\tau(Y)=\mathcal{F}$ iff:

1. $\bigcap \mathcal{F}=\emptyset$,

2. there exists $U \in \mathcal{F}$ such that for every $V \in \mathcal{F}, \bar{U} \backslash V$ is compact,

3. for every $U \in \mathcal{F}$ there exists $V \in \mathcal{F}$ such that $\bar{V} \subset U$.

Proof. It follows from the definition of $\tau(Y)$ and the definition of a locally compact Hausdorff space that those conditions are necessary. We will prove that they are also sufficient. We take $Y:=X \cup\left\{\infty_{Y}\right\}$. A set is open in $Y$ iff it is open in $X$ or it is of the form $U \cup\left\{\infty_{Y}\right\}$ for some $U \in \mathcal{F}$. It follows from (1) and (3) that the topology defined like that is Hausdorff. It remains to show that $Y$ is locally compact. Take $U \in \mathcal{F}$ such that for every $V \in \mathcal{F} \bar{U} \backslash V$ is compact and assume that $\bar{U}$ (closure taken in $Y$ ) is not compact. It follows that there exists a net $\left(x_{\gamma}\right) \subset \bar{U}$ with no convergent subnets. In particular, $\left(x_{\gamma}\right)$ is not convergent to $\infty_{Y}$, so there exists $V_{1}$ a neighborhood of $\infty_{Y}$ and $\left(y_{\gamma}\right)$ a subnet of $\left(x_{\gamma}\right)$ such that $\left(y_{\gamma}\right) \subset \bar{U} \backslash V_{1}$ with no convergent subnets, a contradiction.

We will now provide a characterization for $\mathcal{B}\left(\mathbb{R}^{n}\right)$. To this end, we will need facts about $n$-point Hausdorff compactifications (see [5] or [3, Theorem 6.8]). 
Theorem 2 (Theorem 2.1 in [5]). The following statements concerning a space $X$ are equivalent:

1. X has a N-point compactification.

2. $X$ is locally compact and contains a compact subset $K$ whose complement is the union of $N$ mutually disjoint, open subsets $\left\{G_{i}\right\}_{i=1}^{N}$ such that $K \cup G_{i}$ is not compact for each $i$.

3. $X$ is locally compact and contains a compact subset $K$ whose complement is the union of $N$ mutually disjoint, open subsets $\left\{G_{i}\right\}_{i=1}^{N}$ such that $K \cup G_{i}$ is contained in no compact subset for each $i$.

Using this, we can prove the following facts.

Lemma 1. Let $X$ be a locally compact, noncompact Hausdorff space such that for any $K \subset X$ compact there exists $K_{0}$ compact such that $K \subset K_{0}$ and $X \backslash K_{0}$ has exactly $n$ connected components (for some fixed $n \in \mathbb{N}$ independent of the choice of $K$ ), all of them are open and have noncompact (in $X$ ) closures. Then $X$ has an n-point Hausdorff compactification and does not have an $(n+1)$-point Hausdorff compactification.

Lemma 2. Let $n \in \mathbb{N}$ and $X$ be a Hausdorff topological space that has an n-point Hausdorff compactification and does not have an $(n+1)$-point Hausdorff compactification. Then $X$ is locally compact and $|\mathcal{B}(X)|=2^{n}$.

We will start with Lemma 1.

Proof. Applying the assumption of the lemma to the empty set we get that there exists $n \in \mathbb{N}$ and $K_{0}$ compact such that $X \backslash K_{0}$ has exactly $n$ connected components, let us denote them by $G_{1}, \ldots, G_{n}$. Therefore (by [5]) $X$ has an $n$-point Hausdorff compactification. Suppose that $X$ has an $(n+1)$-Hausdorff compactification. Again by [5], there exist $H_{1}, \ldots, H_{n+1}$ such that $K_{1}:=X \backslash \bigcup_{i=1}^{n+1} H_{i}$ is compact, but for each $i$ the set $K_{1} \cup H_{i}$ is not compact. Applying the assumption of the lemma again, this time to $K_{1}$, we get that there exists a compact set $K_{2}$ such that $K_{1} \subset K_{2}$ and $X \backslash K_{2}$ has $n$ connected components, let us denote them by $V_{1}, \ldots, V_{n}$. Then there exist $i_{0} \in\{1, \ldots, n\}$ and $j_{1}, j_{2} \in\{1, \ldots, n+1\}$ such that $j_{1} \neq j_{2}$ and $H_{i_{0}}$ has nonempty intersection with both $V_{j_{1}}, V_{j_{2}}$, so it cannot be connected, a contradiction.

Now we turn to Lemma 2.

Proof. Since $X$ has an $n$-point Hausdorff compactification, but does not have an $n+1$-point Hausdorff compactification, $\beta X \backslash X$ has exactly $n$ connected components. From the proof of Theorem 1 we know that $|\mathcal{B}(X)|=|\mathcal{A}(X)|$. Each element of $\mathcal{A}(X)$ is a union of some connected components of $\beta X \backslash X$, so $|\mathcal{B}(X)|=|\mathcal{A}(X)|=2^{n}$.

Remark 2. Note that if we assume that if $X$ is a locally compact space such that $|\mathcal{B}(X)|=2^{n}$, we also get that $X$ has an $n$-point Hausdorff compactification and does not have an $(n+1)$-point Hausdorff compactification (see also [3, Theorem 6.32]). 
From the above lemmas we immediately get the following.

\section{Corollary 1.}

- $\mathcal{B}(\mathbb{R})=\left\{\mathbb{R},[-\infty, \infty),(-\infty, \infty], \mathbb{S}^{1}\right\}$.

- $\mathcal{B}\left(\mathbb{R}^{n}\right)=\left\{\mathbb{R}^{n}, \mathbb{S}^{n}\right\}$ for $n \geqslant 2$.

We will now define the end of manifolds, as seen in [2].

Definition 4. Let $L$ be a noncompact, connected manifold. Denote by $\left\{K_{\alpha}\right\}_{\alpha \in \mathcal{K}}$ the family of all compact subsets of $L$. We consider descending chains

$$
U_{\alpha_{1}} \supseteq U_{\alpha_{2}} \supsetneq \cdots \nsupseteq U_{\alpha_{n}} \supseteq \cdots
$$

where each $U_{\alpha_{k}}$ is a connected component of $L \backslash K_{\alpha_{k}}$, has noncompact closure in $L$, satisfies $U_{\alpha_{k}} \supseteq \overline{U_{\alpha_{k+1}}}$ and

$$
\bigcap_{k=1}^{\infty} U_{\alpha_{k}}=\emptyset .
$$

We say that two such chains $\mathcal{U}=\left\{U_{\alpha_{k}}\right\}_{k=1}^{\infty}$ and $\mathcal{V}=\left\{U_{\beta_{k}}\right\}_{k=1}^{\infty}$ are equivalent $(\mathcal{U} \sim \mathcal{V})$ if for each $k \geqslant 1$ there is $n>k$ such that $U_{\alpha_{k}} \supset V_{\beta_{n}}$ and $V_{\beta_{k}} \supset U_{\alpha_{n}}$. It is easy to check that $\sim$ is an equivalence relation. If

$$
\mathcal{U}=\left\{U_{\alpha_{1}} \supseteq U_{\alpha_{2}} \supseteq \cdots \supseteq U_{\alpha_{n}} \supseteq \cdots\right\}
$$

is as above, we call its equivalence class under $\sim$ an end of $L$.

\section{Corollary 2.}

If $L$ is a noncompact, connected, second countable manifold with $n$ ends, $n<\infty$, then $|\mathcal{B}(L)|=2^{n}$.

Proof. Let

$$
\begin{gathered}
\mathcal{U}_{1}=\left\{U_{\alpha_{1}}^{1} \supseteq U_{\alpha_{2}}^{1} \supseteq \cdots\right\} \\
\vdots \\
\mathcal{U}_{n}=\left\{U_{\alpha_{1}}^{n} \supseteq U_{\alpha_{2}}^{n} \supsetneq \cdots\right\}
\end{gathered}
$$

be representatives of the ends of $L$.

For every $k \in\{1,2, \ldots\}, l \in\{1,2, \ldots, n\}$ let $K_{\alpha_{k}}^{l}$ be a compact set such that $U_{\alpha_{k}}^{l}$ is a connected component of $L \backslash K_{\alpha_{k}}^{l}$. We will show that by taking subsequences of $\mathcal{U}_{2}, \ldots, \mathcal{U}_{n}$ we can assume that $U_{\alpha_{k}}^{l_{2}} \subset L \backslash K_{\alpha_{k}}^{l_{1}}$ for every $l_{2}>l_{1}$ (note that a subsequence of a representative of an end is a representative of the same end).

Consider $K_{\alpha_{1}}^{1}$. Then $\left\{L \backslash \overline{U_{\alpha_{1}}^{2}}, L \backslash \overline{U_{\alpha_{2}}^{2}}, \ldots\right\}$ is an open cover of $K_{\alpha_{1}}^{1}$ so there exists $N_{1}>0$ such that $K_{\alpha_{1}}^{1} \subset L \backslash \overline{U_{\alpha_{N_{1}}}^{2}} \subset L \backslash U_{\alpha_{N_{1}}}^{2}$. Therefore $U_{\alpha_{N_{1}}}^{2} \subset L \backslash K_{\alpha_{1}}^{1}$. Similarly, for each $m>1$, we can define $N_{m}>N_{m-1}$ such that $U_{\alpha_{N_{m}}}^{2} \subset L \backslash K_{\alpha_{m}}^{1}$. Replacing $U_{\alpha_{m}}^{2}$ by $U_{\alpha_{N_{m}}}^{2}$ for each $m>0$ we get a subsequence we want for $\mathcal{U}_{2}$. Now we proceed similarly for $\mathcal{U}_{3}, \ldots, \mathcal{U}_{n}$. 
We will now show that by again taking subsequences we can assume that for every $l_{1} \neq l_{2}$ we have $U_{\alpha_{1}}^{l_{1}} \cap U_{\alpha_{1}}^{l_{2}}=\emptyset$. Assume the contrary. Then, without loss of generality, for each $k>0$ we have $U_{\alpha_{k}}^{1} \cap U_{\alpha_{k}}^{2} \neq \emptyset$. Since $U_{\alpha_{k}}^{2} \subset L \backslash K_{\alpha_{k}}^{1}$, the set $U_{\alpha_{k}}^{2}$ is connected, $U_{\alpha_{k}}^{1}$ is a connected component of $L \backslash K_{\alpha_{k}}^{1}$ and $U_{\alpha_{k}}^{1} \cap U_{\alpha_{k}}^{2} \neq \emptyset$, it follows that $U_{\alpha_{k}}^{2} \subset U_{\alpha_{k}}^{1}$ for each $k>0$. Now consider $K_{\alpha_{k}}^{2}$. As before, there exists $N_{k}>k$ such that $K_{\alpha_{k}}^{2} \subset L \backslash U_{\alpha_{N_{k}}}^{1}$. It follows that $U_{\alpha_{N_{k}}}^{1} \subset L \backslash K_{\alpha_{k}}^{2}$. If $U_{\alpha_{N_{k}}}^{1} \not \subset U_{\alpha_{k}}^{2}$ then $U_{\alpha_{N_{k}}}^{1} \cap U_{\alpha_{k}}^{2}=\emptyset$, so $U_{\alpha_{N_{k}}}^{1} \cap U_{\alpha_{N_{k}}}^{2}=\emptyset$ (since $U_{\alpha_{N_{k}}}^{2} \subset U_{\alpha_{k}}^{2}$ ). Therefore $U_{\alpha_{N_{k}}}^{1} \subset U_{\alpha_{k}}^{2}$ and so $\mathcal{U}_{1}$ and $\mathcal{U}_{2}$ are representatives of the same end, a contradiction.

Now our aim is to use Lemmas 1 and 2, which will end the proof. To this end, we will construct a family of compact sets $\left\{K_{j}\right\}_{j=1}^{\infty}$. We will need some properties of manifolds, namely that a second countable manifold is metrizable and that the one-point compactification of a connected manifold is locally connected (see [4] or [6, page 104]). Let $\omega L=L \cup\{\infty\}$ be the one-point compactification of $L$. Since $L$ is second countable we can choose a countable basis of its topology $\mathcal{B}=\left\{B_{1}, B_{2}, B_{3}, \ldots\right\}$ consisting of open sets with compact closures. Take $A_{1}:=K_{\alpha_{1}}^{1} \cup \ldots \cup K_{\alpha_{1}}^{n} \cup \overline{B_{1}}$. Let $K_{1}$ be a compact superset of $A_{1}$ such that $\omega L \backslash K_{1}$ is connected (it exists because $\omega L$ is locally connected). Note that connected components of $L \backslash K_{1}$ are all open and have noncompact (in $L$ ) closures (because $\infty$ is in the closure taken in $\omega L$ of every one of them). Again, because $L$ is locally compact we can take an open set $A_{2}$ with compact closure such that $K_{1} \cup \overline{B_{2}} \subset A_{2}$. Let $K_{2}$ be a compact superset of $A_{2}$ such that $\omega L \backslash K_{2}$ is connected. As before, all connected components of $L \backslash K_{2}$ are open and have noncompact (in $L$ ) closures. Moreover, each of them is contained together with its closure in some connected component of $L \backslash K_{1}$. Note that since $\omega L \backslash K_{2}$ has non-empty intersection with every connected component of $L \backslash K_{1}$ (because $\infty$ is in the closure taken in $\omega L$ of every one of them), for every connected component of $L \backslash K_{1}$ there is at least one connected component of $L \backslash K_{2}$ contained in it. Continuing in this manner, we get $\left\{K_{j}\right\}_{j=1}^{\infty}$. Note that $K_{j}$ is contained in the interior of $K_{j+1}$ for each $j \geqslant 1$ and $\bigcup_{j=1}^{\infty} K_{j}=L$. Moreover, when $j$ increases the number of connected components of $L \backslash K_{j}$ either increases or stays the same. Consider a connected component $U_{1}$ of $L \backslash K_{1}$. We want to show that $U_{1} \cap U_{\alpha_{1}}^{i} \neq \emptyset$ for some $i$. Indeed, otherwise by choosing a connected component $U_{2}$ of $U_{1} \backslash K_{2}$, then a connected $U_{3}$ of $U_{2} \backslash K_{3}$ etc. we would get a representative of an end that is not among $\mathcal{U}_{1}, \ldots, \mathcal{U}_{n}$, a contradiction. Suppose that $U_{1} \cap U_{\alpha_{1}}^{1} \neq \emptyset$. Since $K_{\alpha_{1}}^{1} \subset K_{1}$ and $U_{\alpha_{1}}^{1}, U_{1}$ are connected components of their complements we get $U_{1} \subset U_{\alpha_{1}}^{1}$. The sets $U_{\alpha_{1}}^{i}$ are pairwise disjoint, so $L \backslash K_{1}$ has at least $n$ connected components. Moreover, the number of connected components of $L \backslash K_{j}$ cannot increase past $n$ for any $j$. Indeed, if we had at least $n+1$ connected components of $L \backslash K_{j}$ for some $j$, we could construct at least $n+1$ different ends (similarly as before) which again contradicts the fast that $\mathcal{U}_{1}, \ldots, \mathcal{U}_{n}$ are all of the ends in $L$. Lemma 1 ends the proof.

From this and Remark 2 we also get the following.

Corollary 3. If $L$ is a noncompact, connected, second countable manifold with $n$ ends, $n<\infty$, then $L$ has an $n$-point Hausdorff compactification and does not have an $(n+1)$-point Hausdorff compactification. 


\section{Acknowledgments}

I would like to thank the referees for their valuable remarks, which helped to simplify some proofs and suggestions that greatly improved the paper.

\section{References}

[1] P. Alexandroff, Über die Metrisation der im Kleinen kompakten topologischen Räume, Math. Ann. 92 (1924) 294-301.

[2] A. Candel, L. Conlon, Foliations I, Amer. Math. Soc., 1999.

[3] R. Chandler, Hausdorff Compactifications, Marcel Dekker, New York, 1976.

[4] J. De Groot, R.H. McDowell, Locally connected spaces and their compactifications, Illinois J. Math. 11 Issue 3 (1967) 353-364.

[5] K.D. Magill Jr., N-point compactifications, Am. Math. Mon. 72 (1965) 1075-1081.

[6] R.L. Wilder, Topology of manifolds, Amer. Math. Soc. Colloquium Publications 42 (1949).

DOI: $10.7862 /$ rf.2020.8

\section{Artur Polański}

email: artur.polanski@uj.edu.pl

ORCID: 0000-0002-3302-2551

Department of Functional Analysis

Institute of Mathematics of the Jagiellonian University

POLAND 